\title{
Geoquímica Orgânica de Folhelhos da Formação Pojuca, Bacia do Recôncavo, Brasil
}

\author{
Organic Geochemistry of the Pojuca Formation's Shales, Recôncavo Basin, Brazil
}

\section{Flávia Lima e Cima Miranda ${ }^{1}$ (D), José Roberto Cerqueira ${ }^{1}$ (), Diego Nery do Amaral ${ }^{1}$ (i), Neila Caldas Abreu ${ }^{1}$ (1), Vitória Costa Meirelles Góes ${ }^{1}$ (), Ayana Souza da Silva ${ }^{1}$ (1),

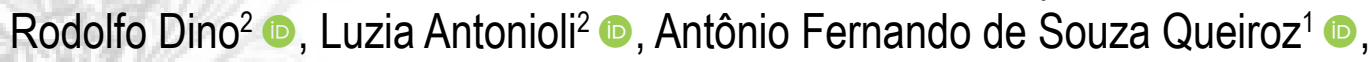 Luiz Carlos Lobato dos Santos ${ }^{1}$ [1] \& Karina Santos Garcia ${ }^{1}$}

1Universidade Federal da Bahia, Instituto de Geociências, Programa de Pós-Graduação em Geoquímica: Petróleo e Meio Ambiente, Salvador, BA, Brasil

${ }^{2}$ Universidade Estadual do Rio de Janeiro, Departamento de Estratigrafia e Paleontologia, Faculdade de Geologia,

Rio de Janeiro, RJ, Brasil

E-mails: cimaflavia@gmail.com; jrkcerq@gmail.com; d.nery9@gmail.com; neila86.nc@gmail.com; vitoriacostameirelles@gmail.com; ayanatrindade@gmail.com; dino@uerj.br; luziaa@uerj.br; queiroz@ufba.br; Iclsantos@ufba.br; karina.ksg4@gmail.com

\section{Resumo}

A bacia sedimentar do Recôncavo, localizada no estado da Bahia, desde a descoberta de petróleo, no final dos anos 30 em Lobato, tem sido intensamente explorada. Atualmente, após 80 anos de exploração ininterrupta é considerada como uma bacia madura. Contudo, ainda é objeto de pesquisas exploratórias e de estudos de cunho acadêmico. Constitui parte integrante do sistema de riftes intracontinentais Recôncavo-Tucano-Jatobá, e seus sistemas petrolíferos possuem em comum a Formação Candeias como rocha geradora principal, depositada na sequência sin-rifte, de idade eocretácea (Berriasiano). Entretanto, avalia-se que a Bacia do Recôncavo é portadora de outros níveis de folhelhos com potencial gerador de hidrocarbonetos; sendo que a própria ANP considera que a Formação Pojuca também apresenta alto potencial gerador, ponderando que a mesma ocorre, em boa parte da bacia, fora da janela de geração. Assim, este trabalho objetiva avaliar a potencialidade para geração de hidrocarbonetos, o estágio de maturação térmica, e o paleoambiente deposicional dos folhelhos da Formação Pojuca, depositadas também na fase sin-rifte, porém de idade relativamente mais jovem (Hauteriviano-Barremiano) que a Formação Candeias. Foram coletadas 48 amostras de folhelhos de um testemunho cortado pelo poço 9-FBA-79-BA, próximo à cidade de Aramari, no estado da Bahia. Os teores de carbono orgânico total (COT) variaram de 1,24 a 4,85 \% e os resultados da pirólise Rock-Eval indicam querogênio predominantemente dos tipos I/II, potencial gerador de hidrocarbonetos (S2) variando de pobre a excelente $(1,26$ a $26,56 \mathrm{mg} \mathrm{HC} / \mathrm{g}$ rocha), baixa a boa concentração de hidrocarbonetos livres (S1) e temperatura máxima (Tmax) da pirólise Rock-Eval que sugere imaturidade termal das amostras $\left(<440^{\circ} \mathrm{C}\right)$. Através da análise de isótopos estáveis de carbono (-33,83\%o a -21,54\%o), foi possível inferir que o paleoambiente deposicional da Formação Pojuca foi lacustre com variações na salinidade da água.

Palavras-Chave: Pirólise Rock-Eval; Isótopos Estáveis de Carbono Orgânico; Paleoambiente deposicional

\begin{abstract}
Located in the Bahia State, the Recôncavo basin has been intensively explored since the first oil discovery, in the late 30 s, at Lobato village. Currently, it is considered a mature basin after 80 years of uninterrupted exploration. However, it is still the subject of exploratory research and academic studies. It is an integral part of the Recôncavo-Tucano-Jatobá intracontinental rift system. Its oil systems have in common the Candeias Formation as the principal source rock, deposited in the syn-rift sequence, of eocretaceous age (Berriasiano). However, it is estimated that the Recôncavo Basin has other levels of shales with potential for generating hydrocarbons; and the ANP itself considers that the Pojuca Formation also has a high generating potential considering that it occurs, in large part of the basin, outside the generation window. Therefore, this work aims to evaluate the potential of hydrocarbon generation, thermal maturation, and the depositional paleoenvironment of Pojuca Formation shales also deposited in the relatively younger (Hauterivian) syn-rift phase than the Candeias Formation. Forty-eight shale core samples were collected from the well 9-FBA-79-BA, near the Aramari city, in the Bahia State. The total organic carbon (TOC) levels ranged from 1.24 to $4.85 \%$, and results of Rock-Eval pyrolysis indicated kerogen types I / II mainly, hydrocarbon generator potential (S2) ranging from poor to excellent (1, 26 to $26.56 \mathrm{mg} \mathrm{HC} / \mathrm{g}$ rock), low to good concentration of free hydrocarbons (S1) and maximum temperature (Tmax) of the Rock-Eval pyrolysis that suggests thermal immaturity of the samples $\left(<440^{\circ} \mathrm{C}\right)$. Through the stable carbon isotopes analysis $(-33.83 \%$ o to $-21.54 \%$ ), it was possible to infer that depositional paleoenvironment from the Pojuca Formation was lacustrine with variations in water salinity.
\end{abstract}

Keywords: Rock-Eval Pyrolysis; Stable Isotopes of Organic Carbon; Depositional paleoenvironment 


\section{Introdução}

ABacia do Recôncavo foi a primeira bacia sedimentar petrolífera brasileira a ser explorada economicamente na década de 1930 (Figueiredo et al. 1994). A exploração de óleo na localidade de Lobato culminou com a descoberta de cerca de 80 campos de óleo e gás, com mais de 5000 poços perfurados (Magnavita, Silva \& Sanches 2005). Tal bacia retrata um rifte intracontinental abortado, preenchido por sedimentos continentais e se caracteriza por uma arquitetura assimétrica de grábens e horts gerada durante o desmembramento do Gondwana, entre o Jurássico tardio e o Cretáceo inicial (Santos 2005; Silva, Caixeta \& Milhomem 2007).

Localizada no centro-leste do estado da Bahia, na porção Nordeste do Brasil, a bacia apresenta uma forma alongada NW-SE (Silva, Caixeta \& Milhomem 2007) e encontra-se delimitada ao norte pelo Alto de Aporá (que a separa da Bacia do Tucano), a leste pelo sistema de falhas de Salvador, ao sul pelos sistemas de falhas da Barra e a oeste pela falha de Maragogipe e Inhambupe (Balbinot \& Kalkreuth 2010; Portela 2012).

O evento tectônico de separação continental, foi o principal responsável pela abertura do sistema RecôncavoTucano-Jatobá. O preenchimento sedimentar da bacia do Recôncavo, na sua fase sin-rifte, ocorreu inicialmente com a deposição de folhelhos e turbiditos do Membro Gomo (Formação Candeias). A princípio, esta deposição se deu em lago restrito e posteriormente, pela intensa atividade tectônica, esse lago foi ampliado durante a deposição da Formação Maracangalha. A diminuição contínua da taxa de subsidência possibilitou que deltas progradassem por meio das plataformas existentes, ocupando os depocentros com arenitos deltaicos das Formações Marfim e Pojuca (Magnavita, 1992; Portela et al. 2016).

Estudos geoquímicos anteriores (Gaglianone \& Trindade, 1988; Figueiredo et al. 1994; Mello et al. 1994) identificaram que a Formação Candeias é a principal rocha geradora da Bacia do Recôncavo, o que não desconsidera que nesta bacia existem outras regiões menos exploradas com outros níveis de folhelhos potencialmente geradores de hidrocarbonetos. Para tal investigação a geoquímica orgânica se torna fundamental para determinar a origem, migração, acumulação, exploração e produção do petróleo (óleo ou gás) (Hunt 1996).

O presente estudo enfoca a análise da geoquímica orgânica da Formação Pojuca na Bacia do Recôncavo, permitindo inferências sobre o potencial gerador, maturação térmica da matéria orgânica e o paleoambiente deposicional. Para alcançar tal objetivo, estudos foram realizados em amostras de folhelhos do testemunho de um poço perfurado na porção NW da Bacia do Recôncavo.

\section{Metodologia}

\subsection{Amostragem}

Foram utilizadas 48 amostras compreendidas no intervalo 31,10 a 98,80 m, de um furo de sondagem rasa, do poço 9-FBA-79-BA (Figura 1), com testemunhagem contínua na Formação Pojuca, Bacia do Recôncavo. Localizado na porção NE do estado da Bahia, próximo a cidade de Aramari, na borda noroeste da bacia, em região próxima ao limite com a Bacia do Tucano, este poço foi perfurado nos anos 80 pela antiga Petromisa, visando a prospecção de sulfetos e cujo acervo de dados hoje se encontra sob os cuidados da Petrobras.

As amostras foram cedidas pela Faculdade de Geologia da Universidade do Estado do Rio de Janeiro (UERJ), e encaminhadas ao Laboratório LEPETRO Excelência em Geoquímica: Petróleo, Energia e Meio Ambiente no Instituto de Geociências (IGEO) pertencente à Universidade Federal da Bahia (UFBA) para as análises de geoquímica orgânica.

\subsection{Análises Geoquímicas}

As análises realizadas foram Pirólise Rock-Eval, resíduo insolúvel $(\mathrm{RI})$ e isótopos estáveis de carbono orgânico, sendo os dados de carbono orgânico total (COT) obtidos através do Rock-Eval. Inicialmente, as amostras foram tratadas para retirada de possíveis impurezas (raízes, fragmentos de tecidos e outros), trituradas com ajuda de um moinho de esfera planetário (Retsch $\mathrm{GmbH}$, modelo PM 400) e peneiradas à 80 mesh.

Para Pirólise Rock-Eval, foram utilizadas cerca de $100 \mathrm{mg}$ de cada amostra pulverizada, peneiradas à $0,177 \mathrm{~mm}$ e encapsuladas, onde foram submetidas à temperatura entre $100^{\circ}$ e $850^{\circ} \mathrm{C}$, a uma taxa de aumento de $25^{\circ} \mathrm{C}$ por minuto (Lafargue, Marquis \& Pillot1998) e analisadas no equipamento Rock-Eval 6 (Espitalié et al. 1977). Para análise dos resultados obtidos foram confeccionados gráficos binários, segundo a classificação de Peters \&Cassa (1994).

Na determinação do resíduo insolúvel, foram pesadas cerca de $1 \mathrm{~g}$ de cada amostra pulverizada, e submetidas a uma reação com uma solução de ácido clorídrico $(\mathrm{HCl})$ aquoso $1 \mathrm{~mol} \mathrm{~L} \mathrm{~L}^{-1}$, para a remoção da fração de carbonato (descarbonatação). As amostras foram então lavadas com água quente destilada e secas no forno a aproximadamente $80{ }^{\circ} \mathrm{C}$ até que o peso constante fosse obtido. Após este processo, temos o resultado de RI, definido como a fração da amostra não eliminada pelo tratamento ácido (fração siliciclástica) (Hedges \& Stern 1984). 


\section{MAPA DE LOCALIZAÇÃO}
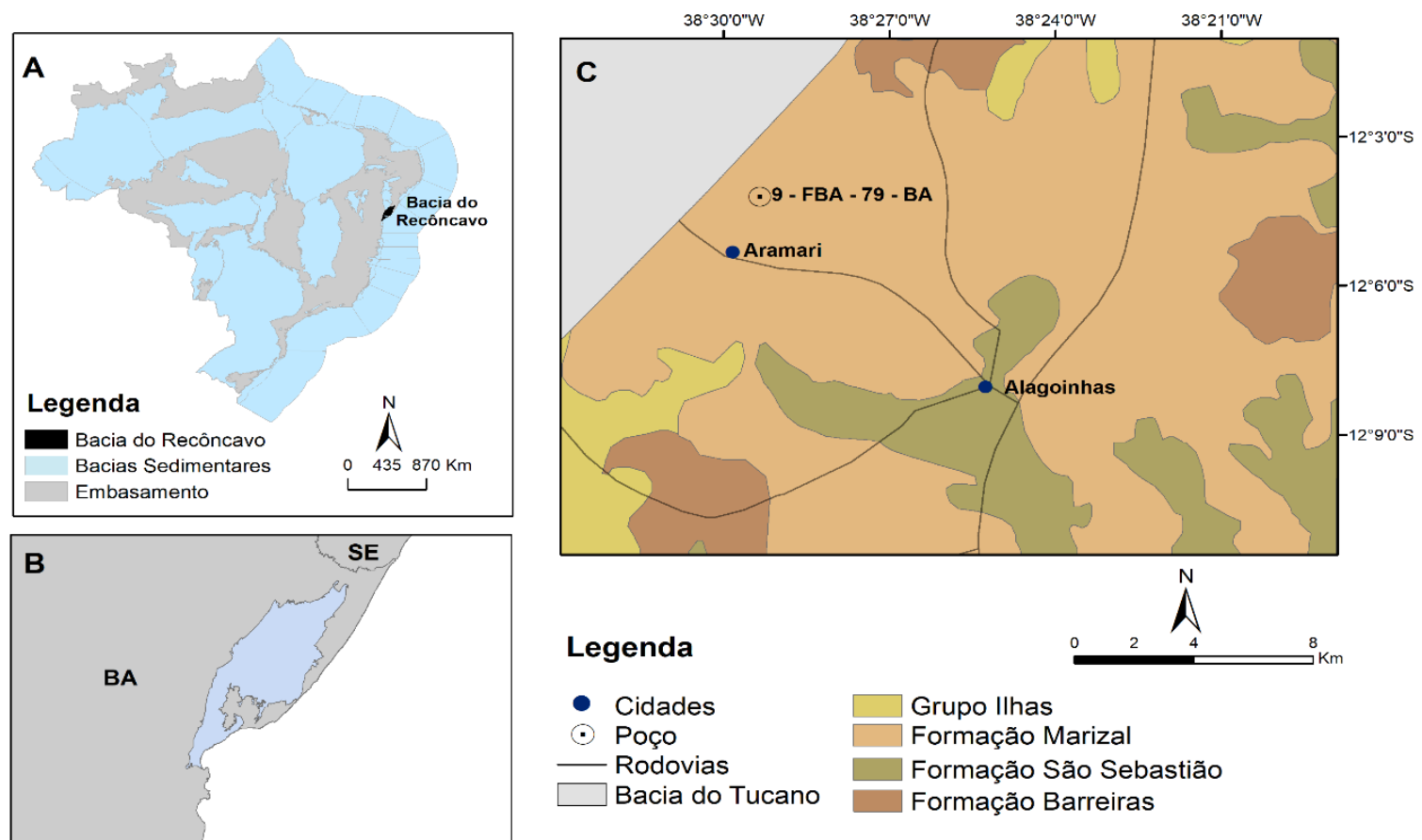

Figura 1 A. Localização da Bacia do Recôncavo no território brasileiro; B. Estados que a delimitam a Bacia do Recôncavo; C. Mapa de localização do poço 9-FBA-79-BA da Formação Pojuca, Bacia do Recôncavo, indicando unidades geológicas (adaptado CPRM, 2006).

Para as análises dos isótopos estáveis de carbono orgânico, foi utilizada cerca de $50 \mathrm{mg}$ de cada amostra descarbonatada e inseridas em cápsulas de estanho, onde foram analisadas em um sistema de Espectrometria de Massas de razão isotópica (IRMS), que identifica e quantifica as moléculas e átomos de uma substância com base na massa dos isótopos, para a determinação da razão $\delta^{13} \mathrm{C}$ (Rodrigues \& Fauth 2013).

Para análise estatística de agrupamento hierárquico de Cluster, foi utilizado o software PAST 4.03, permitindo agrupar as amostras com base nas similaridades e diferenças das características que estes itens possuem (Corrar, Paulo \& Dias Filho 2007).

\section{Resultados e Discussão}

\subsection{Pirólise Rock-Eval}

Os parâmetros quantificados nas amostras do testemunho do poço 9-FBA-79-BA através da pirólise Rock-Eval (COT, S1, S2, IH, IO, Tmáx, IP e RI) estão ilustrados na Tabela 1.

As amostras ao longo do testemunho foram divididas em 3 grupos, com base em uma análise de agrupamento hierárquico das variações ao logo da seção sedimentar. Para isso, foi utilizado o dendrograma resultante da análise de Cluster (Figura 2), utilizando distância euclidiana.

Tabela 1 Parâmetros obtidos na pirólise Rock-Eval, COT= carbono orgânico total (\%), S1= hidrocarbonetos livres (mg HC/g rocha), S2= potencial de geração de hidrocarbonetos ( $\mathrm{mg} \mathrm{HC} / \mathrm{g}$ rocha), Tmáx= temperatura máxima $\left({ }^{\circ} \mathrm{C}\right), \mathrm{IH}=$ índice de hidrogênio $(\mathrm{mg} \mathrm{HC} / \mathrm{g}$ $\mathrm{COT}$ ), IO= índice de oxigênio ( $\mathrm{mg} \mathrm{CO}_{2} / \mathrm{g} \mathrm{COT}$ ), IP = índice de produção, RI= Resíduo insolúvel (\%) nas amostras do poço 9-FBA-79BA, Formação Pojuca, Bacia do Recôncavo.

\begin{tabular}{|c|c|c|c|c|c|c|c|c|}
\hline $\begin{array}{l}\text { Amostra } \\
\text { (Prof. m) }\end{array}$ & $\begin{array}{c}\text { COT } \\
(\%)\end{array}$ & $\begin{array}{c}\mathrm{S1} \\
\text { (mg HC/g rocha) }\end{array}$ & $\begin{array}{c}\mathrm{S2} \\
\text { (mg HC/g rocha) }\end{array}$ & Tmáx. $\left({ }^{\circ} \mathrm{C}\right)$ & $\begin{array}{c}\mathrm{IH} \\
\text { (mg HC/g COT) }\end{array}$ & $\begin{array}{c}10 \\
(\mathrm{mg} \mathrm{CO} / \mathrm{g} \mathrm{COT})\end{array}$ & IP & $\begin{array}{r}\text { RI } \\
(\%)\end{array}$ \\
\hline 31,10 & 1,42 & 0,14 & 4,35 & 430 & 306 & 39 & 0,031 & 83,29 \\
\hline 32,30 & 0,91 & 0,07 & 1,56 & 430 & 171 & 38 & 0,043 & 91,10 \\
\hline 33,50 & 1,24 & 0,11 & 3,67 & 429 & 296 & 37 & 0,029 & 79,38 \\
\hline 43,70 & 3,05 & 0,24 & 10,45 & 425 & 343 & 30 & 0,022 & 82,10 \\
\hline
\end{tabular}


Tabela 1 Cont.

\begin{tabular}{|c|c|c|c|c|c|c|c|c|}
\hline $\begin{array}{l}\text { Amostra } \\
\text { (Prof. m) }\end{array}$ & $\begin{array}{l}\text { COT } \\
(\%)\end{array}$ & $\begin{array}{c}\mathrm{S1} \\
\text { (mg HC/g rocha) }\end{array}$ & $\begin{array}{c}\mathrm{S} 2 \\
\text { (mg HC/g rocha) }\end{array}$ & Tmáx. $\left({ }^{\circ} \mathrm{C}\right)$ & $\begin{array}{c}\text { IH } \\
\text { (mg HC/g COT) }\end{array}$ & $\begin{array}{c}\mathrm{IO} \\
(\mathrm{mg} \mathrm{CO} / \mathrm{g} \mathrm{COT})\end{array}$ & IP & $\begin{array}{c}\text { RI } \\
(\%)\end{array}$ \\
\hline 44,30 & 1,40 & 0,19 & 2,72 & 431 & 194 & 39 & 0,065 & 84,59 \\
\hline 52,10 & 1,85 & 0,19 & 4,21 & 429 & 228 & 46 & 0,043 & 77,94 \\
\hline 53,30 & 1,78 & 0,19 & 3,94 & 427 & 221 & 46 & 0,046 & 80,45 \\
\hline 54,20 & 2,06 & 0,21 & 5,72 & 427 & 278 & 43 & 0,035 & 82,54 \\
\hline 56,30 & 2,08 & 0,28 & 4,96 & 428 & 238 & 42 & 0,053 & 83,19 \\
\hline 57,20 & 1,66 & 0,27 & 3,47 & 429 & 209 & 46 & 0,072 & 81,13 \\
\hline 59,30 & 1,71 & 0,17 & 4,05 & 430 & 237 & 49 & 0,040 & 69,23 \\
\hline 60,20 & 2,12 & 0,21 & 5,65 & 429 & 267 & 43 & 0,036 & 59,11 \\
\hline 61,20 & 3,54 & 0,53 & 12,84 & 430 & 363 & 33 & 0,040 & 75,70 \\
\hline 62,10 & 3,23 & 0,35 & 10,56 & 429 & 327 & 30 & 0,032 & 71,86 \\
\hline 62,70 & 2,68 & 0,24 & 9,63 & 428 & 359 & 29 & 0,024 & 53,35 \\
\hline 63,30 & 4,85 & 0,41 & 19,65 & 431 & 405 & 22 & 0,020 & 75,60 \\
\hline 63,60 & 4,01 & 0,36 & 14,69 & 429 & 366 & 29 & 0,024 & 79,88 \\
\hline 63,90 & 4,07 & 0,47 & 15,58 & 429 & 383 & 29 & 0,029 & 85,77 \\
\hline 64,20 & 4,41 & 0,39 & 17,20 & 431 & 390 & 26 & 0,022 & 79,91 \\
\hline 64,50 & 3,36 & 0,36 & 13,22 & 430 & 393 & 31 & 0,027 & 79,80 \\
\hline 65,00 & 3,65 & 0,67 & 14,32 & 432 & 392 & 27 & 0,045 & 76,56 \\
\hline 66,20 & 4,40 & 0,83 & 16,56 & 432 & 376 & 26 & 0,048 & 83,54 \\
\hline 66,80 & 3,45 & 0,38 & 12,40 & 431 & 359 & 26 & 0,030 & 70,64 \\
\hline 67,10 & 4,24 & 1,63 & 15,88 & 432 & 375 & 27 & 0,093 & 84,65 \\
\hline 67,40 & 4,63 & 0,44 & 17,27 & 430 & 373 & 22 & 0,025 & 82,55 \\
\hline 68,00 & 3,12 & 0,46 & 10,55 & 431 & 338 & 31 & 0,042 & 84,83 \\
\hline 68,30 & 3,80 & 0,42 & 14,09 & 431 & 371 & 24 & 0,029 & 87,62 \\
\hline 68,60 & 1,92 & 0,21 & 5,21 & 432 & 271 & 41 & 0,039 & 78,72 \\
\hline 68,90 & 2,06 & 1,47 & 6,18 & 433 & 300 & 35 & 0,192 & 72,37 \\
\hline 73,70 & 4,48 & 0,69 & 24,23 & 436 & 541 & 25 & 0,028 & 79,41 \\
\hline 74,30 & 4,43 & 0,41 & 23,11 & 430 & 522 & 26 & 0,017 & 84,73 \\
\hline 74,60 & 4,60 & 0,87 & 26,26 & 429 & 571 & 27 & 0,032 & 86,11 \\
\hline 75,50 & 2,00 & 1,08 & 6,84 & 431 & 342 & 38 & 0,136 & 55,31 \\
\hline 76,70 & 3,52 & 0,43 & 19,11 & 432 & 543 & 30 & 0,022 & 76,82 \\
\hline 77,60 & 4,57 & 0,86 & 26,43 & 435 & 578 & 22 & 0,032 & 81,56 \\
\hline 78,50 & 2,73 & 0,74 & 11,88 & 429 & 435 & 29 & 0,059 & 73,40 \\
\hline 79,40 & 4,08 & 0,64 & 21,56 & 428 & 528 & 27 & 0,029 & 79,41 \\
\hline 81,80 & 3,83 & 0,81 & 19,35 & 425 & 505 & 35 & 0,040 & 79,19 \\
\hline 82,70 & 1,10 & 0,27 & 2,55 & 430 & 232 & 65 & 0,096 & 77,48 \\
\hline 85,70 & 3,31 & 0,75 & 15,54 & 425 & 469 & 36 & 0,046 & 82,20 \\
\hline 90,80 & 3,32 & 0,56 & 20,43 & 423 & 615 & 28 & 0,027 & 90,77 \\
\hline 91,70 & 2,07 & 1,18 & 10,05 & 426 & 486 & 33 & 0,105 & 81,74 \\
\hline 92,60 & 2,23 & 0,52 & 11,48 & 423 & 515 & 33 & 0,043 & 86,00 \\
\hline 93,50 & 2,75 & 0,31 & 15,37 & 424 & 559 & 30 & 0,020 & 91,15 \\
\hline 94,10 & 1,07 & 0,11 & 2,39 & 433 & 223 & 56 & 0,044 & 82,78 \\
\hline 96,50 & 1,57 & 0,20 & 5,15 & 426 & 328 & 45 & 0,037 & 84,08 \\
\hline 97,90 & 1,69 & 0,52 & 5,24 & 429 & 310 & 53 & 0,090 & 86,55 \\
\hline 98,80 & 1,12 & 0,31 & 3,15 & 429 & 281 & 58 & 0,090 & 81,14 \\
\hline
\end{tabular}




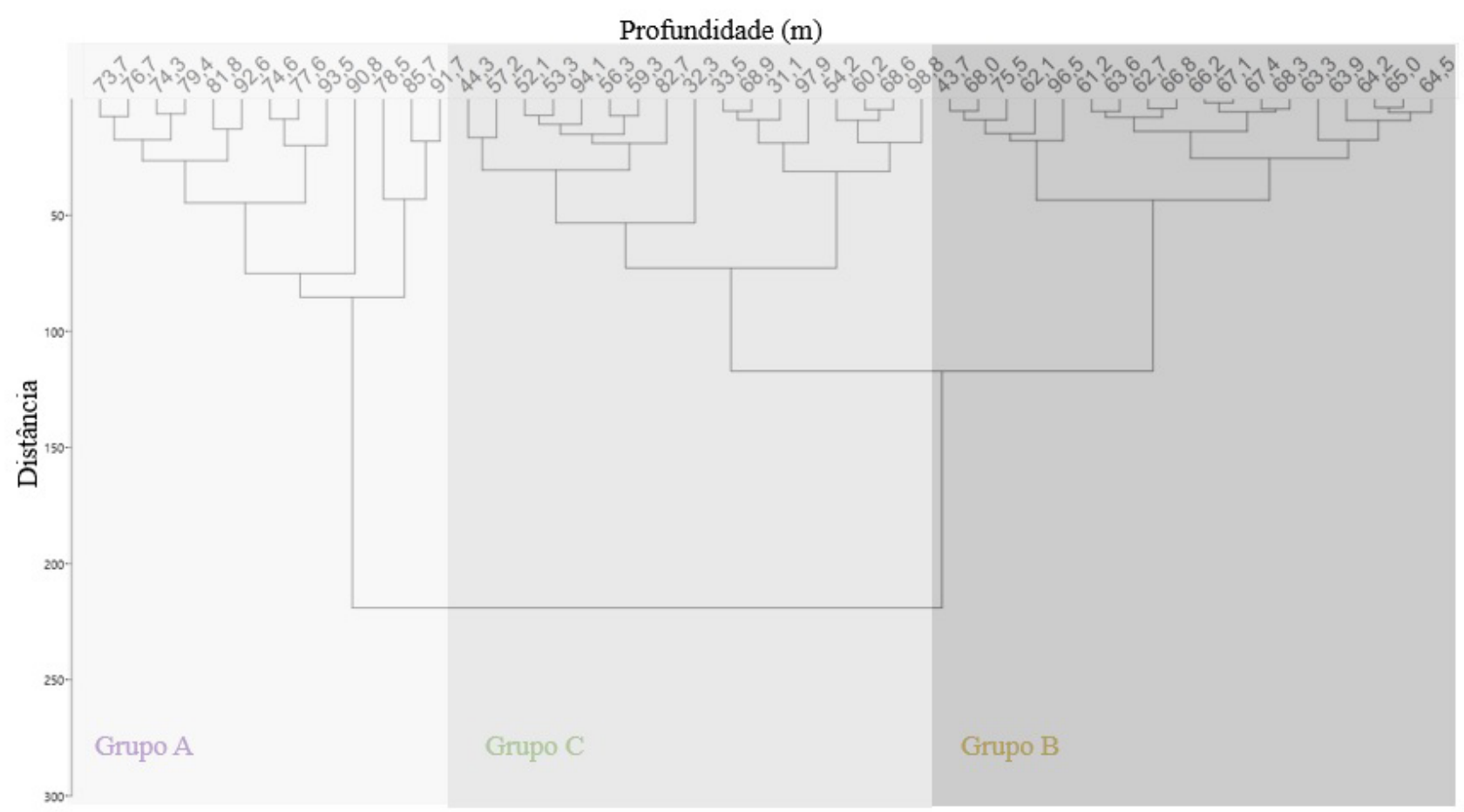

Figura 2 Dendrograma resultante da análise de Cluster para as amostras da Formação Pojuca, Bacia do Recôncavo, através do software PAST 4.03 (distância euclidiana).

O grupo A, de acordo com a classificação de Peters \& Cassa (1994), apresentou valores de COT (2,03-4,57\%) variando de muito alto a excelente, o S1 $(0,31-1,18 \mathrm{mg}$ $\mathrm{HC} / \mathrm{g}$ rocha) sendo classificado de moderado a boa geração natural, o S2 (10,25 - 26,43 mg HC/g rocha) caracterizado por excelente potencial de geração de hidrocarbonetos e o $\mathrm{IH}(435-615 \mathrm{mg} \mathrm{HC} / \mathrm{g}$ COT$)$ sendo indicativo de potencial para geração de óleo.

O grupo B, apresentou valores de COT (1,57$4,85 \%)$ variando de médio a excelente, o S1 (0,2 -1,63 mg $\mathrm{HC} / \mathrm{g}$ rocha) variando de pouco a boa geração natural de hidrocarbonetos, o S2 (5,15- 19,65 mg HC/g rocha) variou de bom a excelente potencial gerador e os valores de $\mathrm{IH}$ (327 - $405 \mathrm{mg} \mathrm{HC} / \mathrm{g} \mathrm{COT)} \mathrm{apresentaram} \mathrm{como} \mathrm{indicativo}$ de potencial para geração de óleo (Peters \& Cassa 1994).

$\mathrm{O}$ grupo $\mathrm{C}$, apresentou valores de COT variando de baixo a alto $(0,91-2,12 \%), \mathrm{S} 1(0,07-0,52 \mathrm{mg} \mathrm{HC} / \mathrm{g}$ rocha) sendo classificado por pouca geração natural, o $\mathrm{S} 2$ (1,56 -6,18 $\mathrm{mg} \mathrm{HC/g}$ rocha) caracterizado por baixo potencial de geração de hidrocarbonetos, o IH (171 - 310 $\mathrm{mg} \mathrm{HC/g} \mathrm{COT)} \mathrm{sendo} \mathrm{indicativo} \mathrm{de} \mathrm{potencial} \mathrm{para} \mathrm{geração}$ de gás e condensado (Peters \& Cassa 1994).

Com base em estudo geoquímico e palinológico dessas mesmas amostras, Portella et al. (2016) individualizaram três zonas de palinofácies em função das proporções de matéria orgânica amorfa, palinomorfos e fitoclastos, e concluíram que a variação dos parâmetros geoquímicos foi resultado das diferentes condições climáticas que proporcionaram períodos de elevação e diminuição do nível do lago.

Para ser uma boa geradora de hidrocarbonetos, a rocha deve possuir altos valores de COT, mas isso não significa que toda matéria orgânica possui a mesma eficiência para geração, uma vez que, a depender do seu tipo, a matéria orgânica pode ou não gerar hidrocarbonetos líquidos e/ou gasosos (Tissot et al. 1974). Dessa forma, a utilização somente do COT como indicador da quantidade de hidrocarbonetos que uma rocha pode gerar não é confiável. Mas ao relacionar esse indicador com o S2, é possível inferir a quantidade matéria orgânica que está presente e quanto hidrogênio está associado a ela. Desse modo, a partir dos parâmetros fornecidos pela pirólise Rock-Eval, é possível concluir que amostras com valores de S2 e COT consistentemente mais altos representam a melhor rocha geradora de hidrocarbonetos (Dembicki Jr 2009) (Figura 3).

Os valores de Tmax em sua maioria, foram inferiores a $440^{\circ} \mathrm{C}$. Para esses valores, deve-se usar a confiabilidade nos valores de $\mathrm{S} 2$ acima de $2 \mathrm{mg} / \mathrm{HC}$ g rocha, portanto, majoritariamente a seção se encontra imatura termicamente 


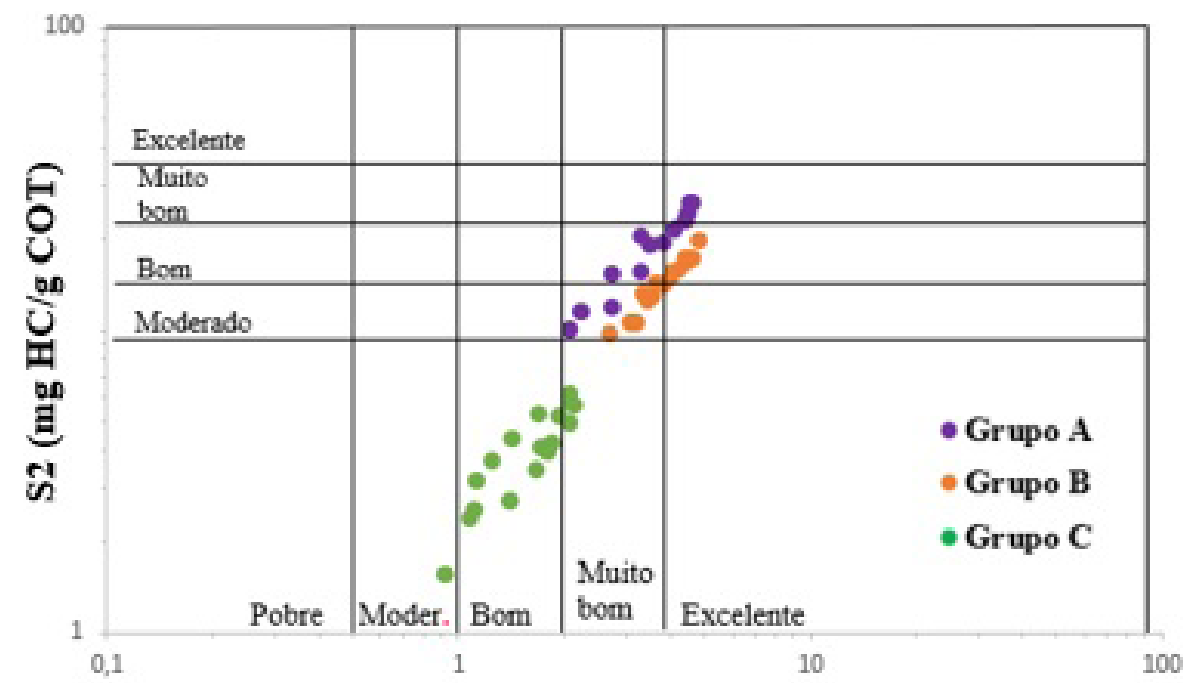

\section{COT $(\%)$}

Figura 3 Classificação do potencial para geração de hidrocarbonetos em função da relação S2 versus COT das amostras da Formação Pojuca, Bacia do Recôncavo (Dembicki Jr. 2009).

para geração de hidrocarbonetos (Peters 1986; Peters \& Moldowan 1993). Apoiado nessa interpretação, os valores de IP foram $<0,1$, sugerindo igualmente, imaturidade térmica (Tissot \& Welte 1984).

Utilizando o diagrama tipo van Krevelen (Tissot \& Welte 1978, 1984), a partir da correlação do IH, que é aproximadamente equivalente à razão atômica $\mathrm{H} / \mathrm{C}$, e do IO que é aproximadamente equivalente à razão atômica O/C (Figura 4), foi possível caracterizar o querogênio presente nos folhelhos da Formação Pojuca como sendo predominantemente dos tipos I/II, derivados de matéria orgânica de boa qualidade para geração de hidrocarbonetos líquidos e gasosos. A maioria dos sedimentos contém misturas de dois ou mais tipos querogênios (Dembicki Jr. 2009), portanto, apesar da classificação do querogênio como sendo predominantemente dos tipos I/II, algumas amostras exibem perfis do tipo II/III, principalmente as do intervalo $\mathrm{C}$.

Convém mencionar que Coutinho (2008), estudando a energia de ativação em amostras de folhelhos geradores da Formação Candeias, verificou que, apesar dos IH e IO classificarem o querogênio como sendo do tipo II, a cinética era característica de querogênio lacustre do tipo I. $\mathrm{O}$ autor concluiu que os valores relativamente mais baixos de $\mathrm{IH}$ fornecidos pela pirólise, eram devidos a oxidação parcial da matéria orgânica no ambiente sedimentar. É provável que isso também tenha ocorrido durante a deposição dos sedimentos da Formação Pojuca, e que a matéria orgânica precursora do querogênio nela presente, tenha sido parcialmente oxidada diminuindo os valores de IH,

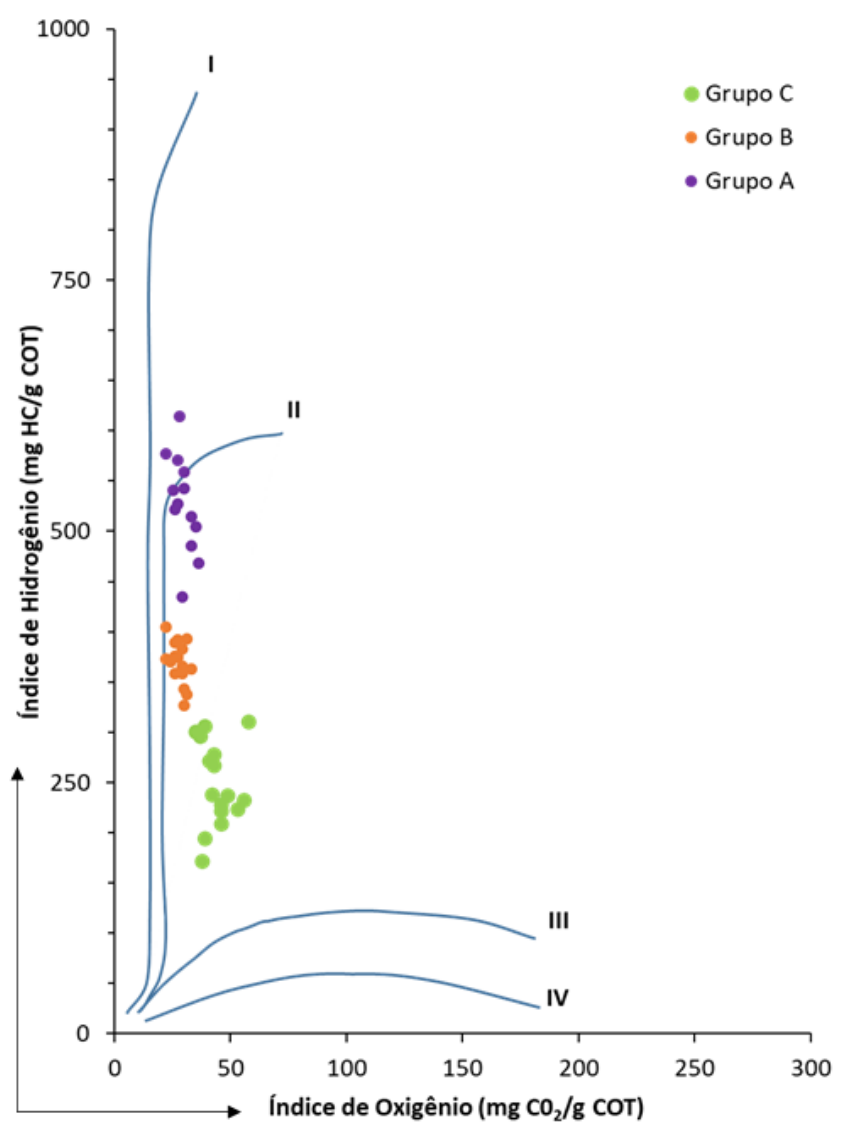

Figura 4 Diagrama do tipo van Krevelen com distribuição das amostras do poço 9-FBA-79-BA em intervalos, Formação Pojuca, Bacia do Recôncavo (Tissot \& Welte 1984; Euzébio et al. 2016). 
levando a uma "falsa" classificação das amostras do grupo A através do diagrama tipo van Krevelen.

No poço estudado, observa-se uma nítida tendência de aumento dos valores do IH para as amostras dos grupos $\mathrm{A}$ e B, o que indica que durante a deposição desse material ocorreu uma melhor preservação da matéria orgânica em ambientes anóxico a disóxico, respectivamente (Figura 5). No grupo C, ocorre uma diminuição nos valores de $\mathrm{IH}$, o que sugere uma mudança no paleoambiente deposicional, relacionada a uma condição mais óxica, e com algum aporte de matéria orgânica continental. Apesar do diagrama IH x RI mostrar que as amostras do grupo C estão no campo de ambiente disóxico, o conjunto de interpretações ao longo deste trabalho, como os valores de IH, IO, S2, COT, sugerem que o ambiente se tornou mais óxico durante o tempo de deposição dos sedimentos representados por essas amostras.

Esta interpretação, se apoia na discussão de Tissot \& Welte (1984), na qual, mudanças no tipo de querogênio dentro de uma mesma formação podem ocorrer devido a variações de fácies.

Alguns autores (Dean, Arthur \& Claypool1986; Routh, Mcdonald \& Grossman 1999; Zambrano, Oliveira \& Ribeiro 2017) usaram a correlação do IH versus COT para inferir a quantidade de entrada de MO terrestre e condições de oxigenação da água em diferentes ambientes deposicionais. Tal análise corrobora a justificativa das variações nas condições (por exemplo, regime de oxigênio, hidrodinâmica no fluxo sedimentar) do ambiente, sugerido pelos valores de IH e COT das amostras (Figura 6). As amostras do grupo B e do grupo A, que apresentaram maiores valores de COT, maiores valores de IH e menores valores IO, apontam melhor preservação e/ou produtividade de matéria orgânica, mostrando deposição em condições anóxicas.

\subsection{Isótopos estáveis de carbono}

Os valores para a razão isotópica $\delta^{13} \mathrm{C}$ do carbono orgânico presente nas amostras de folhelhos refletem a assinatura isotópica dos compostos ali presentes, servindo como registro histórico das contribuições de diferentes fontes de matéria orgânica e das condições físico-químicas do ambiente sedimentar (Pereira 2007).

Observa-se que as amostras apresentaram os valores de $\delta^{13} \mathrm{C}$ variando de $-33,83 \%$ a $-21,54 \%$ (Figura 7), sugerindo fortes alterações nas condições físico-químicas do paleoambiente deposicional. A matéria orgânica presente em ambientes lacustres de água doce é registrada com valores isotópicos menores que $-28 \%$, enriquecida $\mathrm{em}{ }^{12} \mathrm{C}$, enquanto, em ambientes salinos, devido ao enriquecimento relativo em ${ }^{13} \mathrm{C}$, os valores de $\delta^{13} \mathrm{C}$ são maiores que $-28 \%$ (Gaglianone \& Trindade 1988; Mello et al. 1988).

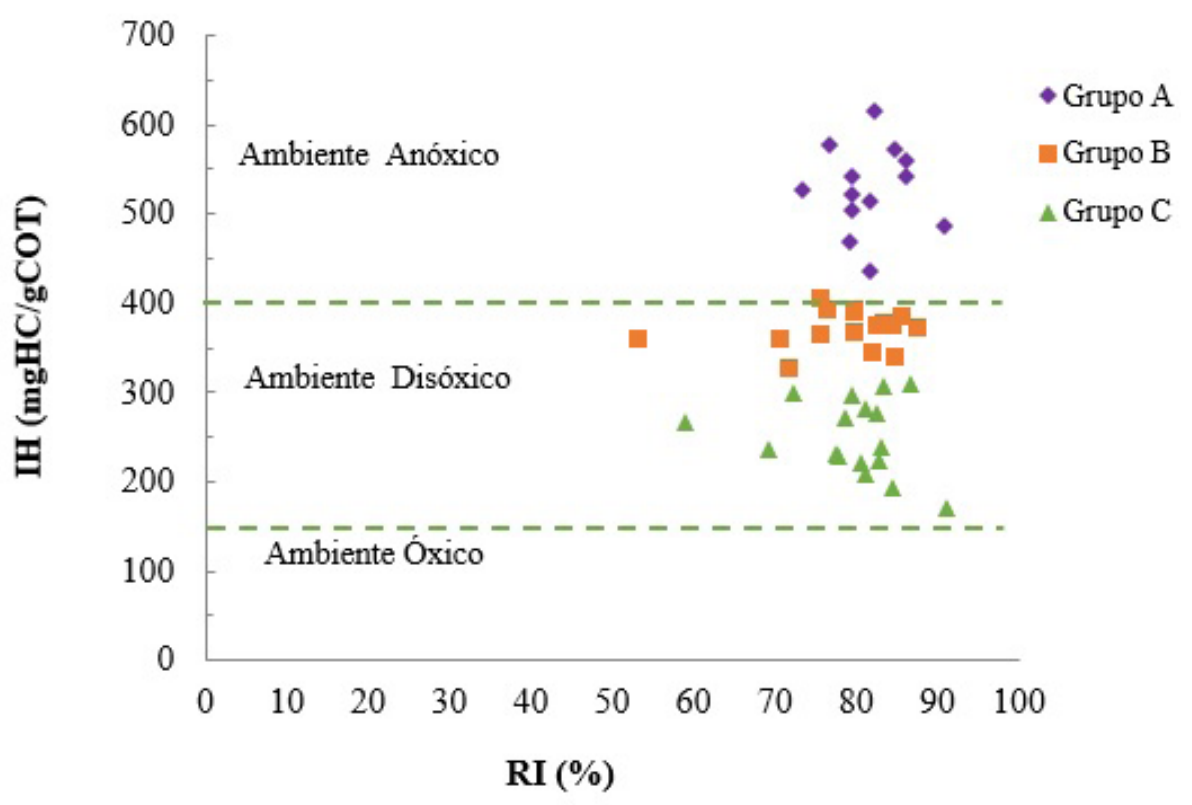

Figura 5 Relação entre os valores de IH e RI para as amostras do poço 9-FBA-79-BA em intervalos, Formação Pojuca, Bacia do Recôncavo (Euzébio et al. 2016). 


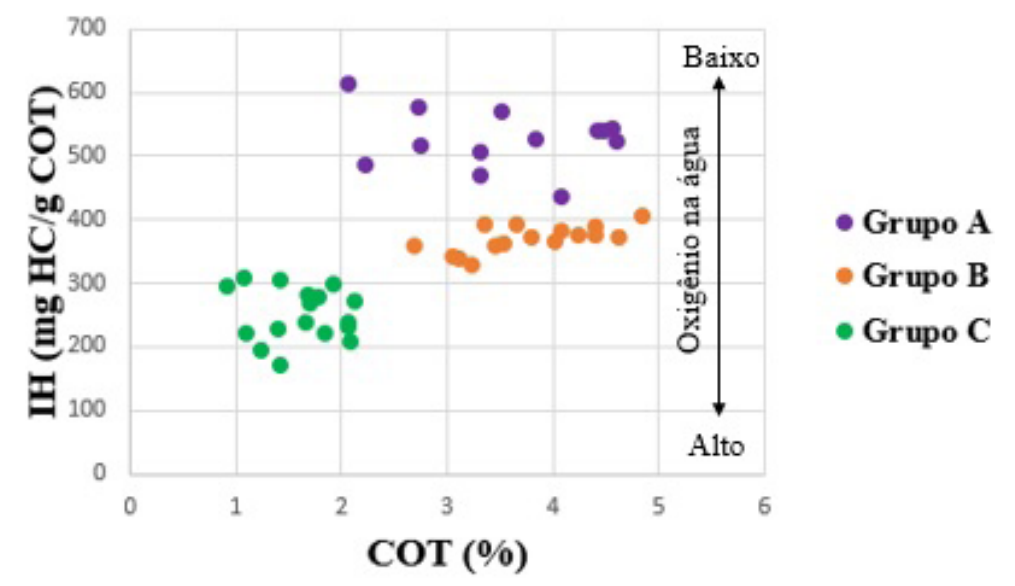

Figura 6 Diagrama do índice de hidrogênio versus carbono orgânico total para as amostras de rocha do poço 9-FBA-79-BA, Formação Pojuca, Bacia do Recôncavo (Modificado de Routh, Mcdonald \& Grossman 1999).

É possível notar que as amostras da base do testemunho apresentaram valores de $\delta^{13} \mathrm{C}$ chegando a $-21,54 \%$, o que é característico de ambiente salino a hipersalino, o que se torna mais evidente ao observar as amostras em relação as suas profundidades (Figura 7). Devido a sua densidade, ambientes salinos não possuem a mesma facilidade de troca de $\mathrm{CO}_{2}$ com a atmosfera que um ambiente de água doce, tornando-o mais enriquecido em $\mathrm{CO}_{2}$ contendo mais ${ }^{13} \mathrm{C}$ (Pereira 2007).

A salinidade elevada, indicada pela positivação dos valores de $\delta^{13} \mathrm{C}$ de algumas amostras, sugere ambiente árido com muita evaporação, e que, possivelmente, em alguns momentos quando da deposição da Formação Pojuca houve aporte de águas salinas na bacia, o que pode estar relacionada a águas conatas, que têm sua origem dentro da própria bacia (presentes em poros de rochas sedimentares durante a deposição e soterramento sedimentar) vinda de locais super pressurizados, e apresentam uma salinidade crescente com a sua profundidade (Beckel 1964). Através da análise de biomarcadores em diferentes óleos da Bacia do Recôncavo, foram identificados compostos específicos de ambientes relativamente salinos (Casilli, et al. 2014).

Como mencionado acima, o aumento de salinidade pode estar refletindo que a deposição desses sedimentos ocorreu durante um clima árido, promovendo uma maior evaporação e diminuição da coluna d'água, tornando o lago mais salino. Santos (2005) realizou estudo dos ciclos flúviodeltaicos-lacustres que caracterizam o Andar Buracica (Barremiano) na Bacia do Recôncavo. Segundo o autor, a progradação flúvio-deltaica é favorecida em períodos úmidos, com maior influxo de água e elevação do nível de base. Após, ocorre redução do nível de base, com a implantação de condições de maior aridez e maiores taxas de evaporação. Folhelhos pretos enriquecidos em carbono orgânico ocupariam uma posição específica neste ciclo, segundo o autor, estando relacionados ao estágio inicial em que se reduz o influxo de água e, consequentemente, o aporte de terrígenos, para implantação de um regime climático com balanço hídrico negativo (aridização), com redução do nível de base.

Portela et al. (2016) também verificaram uma ciclicidade, na qual se alternam depósitos de sistemas lacustres transgressivos e regressivos devido a ocorrência de flutuações periódicas da lâmina d'água como resultado de variações climáticas com reflexos nas proporções das palinofácies analisadas.

Guzzo (1997), verificou deflexões nos valores da razão isotópica $\delta^{13} \mathrm{C}$ e $\delta^{18} \mathrm{O}$ de carbonatos presentes em folhelhos da Formação Pojuca. Apoiado nesses valores, concluiu que as deflexões são devidas a um aumento da produtividade orgânica, em virtude ao maior influxo de água em condições de lago baixo estratificado, por efeito, da evaporação anterior.

Adotando-se os modelos de, Santos (2005) Portella (2016) e Guzzo (1997), as amostras do Grupo A testemunhariam este contexto inicial de queda do nível do lago, sob balanço hídrico negativo, representado por valores menos negativos para a razão $\delta^{13} \mathrm{C}$, indicativa de maior salinidade relativa devido à evaporação da água do lago. As amostras do Grupo C (com valores predominantemente depletados para a razão de $\delta^{13} \mathrm{C}$; predominantemente mais negativos) registrariam um período com maior influxo de água e consequente elevação do nível de base (nível do lago), sob clima mais úmido e maior aporte de constituintes terrígenos. Já as amostras do Grupo B marcariam a transição entre as duas condições, ocorrendo mais influxo de terrígenos e consequente maior oxigenação no lago, em comparação com o Grupo A, porém ainda com boas condições para preservação da matéria (Figura 7). 


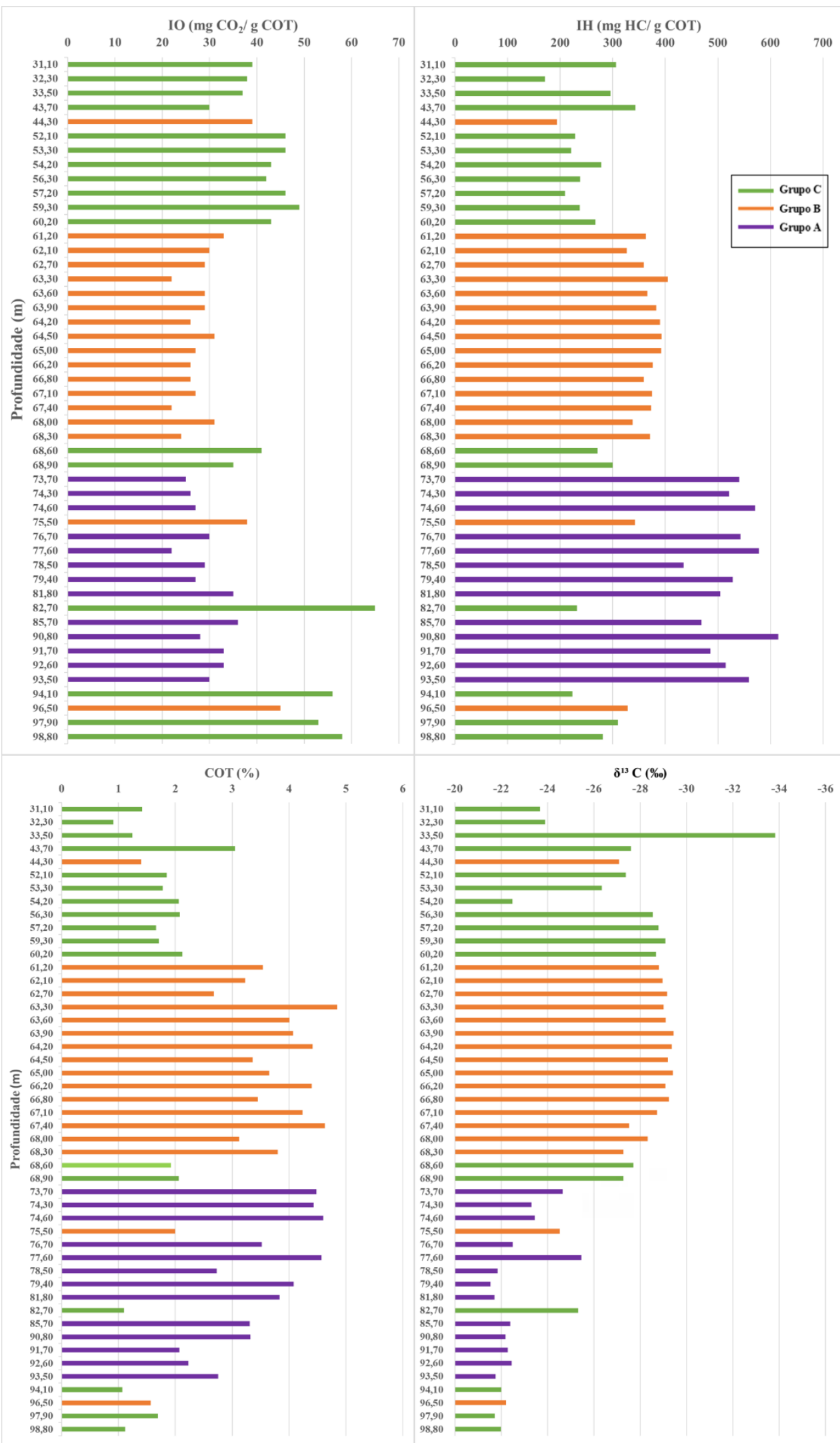

Figura 7 Comportamento dos diferentes parâmetros analisados $\left(\mathrm{COT}, \mathrm{IH}, \mathrm{IO}\right.$ e $\left.\delta^{13} \mathrm{C}\right)$, considerando as amostras da base para o topo, no testemunho do poço 9-FBA-79-BA, Formação Pojuca, Bacia do Recôncavo. 
Tal interpretação está compatível com o proposto por Amaral et al. (2020), onde o autor relata que, ao analisar amostras da Formação Candeias, os folhelhos negros enriquecidos em matéria orgânica estão associados a deposições em clima árido (reduzido influxo de água, com condições propícias à estratificação do corpo d'água, ao desenvolvimento de anoxia de fundo e à consequente preservação da matéria orgânica), enquanto as camadas menos enriquecidas em matéria orgânica estariam relacionadas a deposições em clima úmido (que possibilita maiores descargas fluviais, proporcionando a circulação e consequente oxigenação da massa d'água).

\section{Conclusões}

Com base nos dados de geoquímica orgânica (parâmetros de Pirólise de Rock-Eval e isótopos estáveis de carbono orgânico) das amostras de folhelho da Formação Pojuca do poço 9-FBA-79-BA, na Bacia do Recôncavo, foi possível determinar um excelente potencial gerador, evidenciado pelos valores de COT, S1 e S2. O querogênio presente é predominantemente dos tipos I/II, possivelmente resultante de matéria orgânica amorfa em diferentes níveis de oxidação, e algumas amostras apresentam querogênio do tipo II/III devido algum aporte de matéria orgânica terrígena. Tmax e IP indicam que as amostras se encontram imaturas termicamente para a geração de hidrocarbonetos, sem efeitos térmicos importantes devido a insuficiência de soterramento

$\mathrm{O}$ perfil de $\delta^{13} \mathrm{C}$, variável ao longo do testemunho estudado, sugere diferentes condições físico-químicas no paleoambiente deposicional, tornando possível a interpretação de que a matéria orgânica é característica de um ambiente lacustre com variação de salinidade, podendo estar relacionada com a deposição dos sedimentos em um clima árido com elevada evaporação, possivelmente, com contribuição de presença de águas conatas.

\section{Agradecimentos}

Os autores gostariam de agradecer ao Programa de Pós-Graduação em Geoquímica: Petróleo e Meio Ambiente (POSPETRO) da Universidade Federal da Bahia (UFBA) e à Faculdade de Geologia da Universidade do Estado do Rio de Janeiro (UERJ), por todo o apoio durante a elaboração deste artigo.

Esta pesquisa foi realizada em associação com o projeto em andamento de $\mathrm{P} \& \mathrm{D}$ registrado como ANP No20075-8, "Project Petroleum Systems Research in Brazilian Sedimentary Basins" (UFBA / Shell Brasil / ANP) - título de projeto da ANP (Pesquisas em Sistemas Petrolíferos de Bacias Sedimentares Brasileiras), patrocinado pela Shell Brasil sob a aplicação da P \& D da ANP como "Compromisso de Investimentos com Pesquisa e Desenvolvimento"; e a Coordenação de Aperfeiçoamento de Pessoal de Nível Superior (CAPES, Brasília, Brasil) pela bolsa de estudo - código de financiamento 001 .

\section{Referências}

Amaral, D.N., Cerqueira, J.R., Andrade, C.L.N., Ribeiro, H.J.P.S., Garcia, K.S., Miranda, F.L.C., Oliveira, O.M., Queiroz, A.F. \& Santos, L.C.L. 2020, 'Paleoenvironmental characterization of a Lower Cretaceous section of the Recôncavo Basin, Bahia, Brazil', Brazilian Journal of Geology, vol. 50, no. 3, pp. 1-11. https://doi.org/10.1590/2317-4889202020190058

Balbinot, M. \& Kalkreuth, W. 2010, 'Organic geochemistry and petrology of the Gomo Member, Recôncavo Basin', Brazil. International Journal of Coal Geology, vol. 84, no. 3-4, pp. 286-92.https://doi.org/10.1016/j.coal.2010.09.008

Beckel, J. 1964, 'Depositos de sulfetos sinsedimentares: um processo exalativo de salmouras conatas metalífera', Revista Brasileira de Geociências, vol. 14, no. 4, pp. 183-93. http:// bjg.siteoficial.ws/1984/n4/beckel.pdf

Casilli, A., Silva, R.C., Laakia, J., Oliveira, C.J.F., Ferreira, A.A., Loureiro, M.R.B., Azevedo, D.A. \& Neto, F.R.A. 2014, 'High resolution molecular organic geochemistry assessment of Brazilian lacustrine crude oils', Organic Geochemistry, vol. 68, no. 61-70. https://doi.org/10.1016/j.orggeochem.2014.01.00

Coutinho, L.F.C. 2008, 'Análise do balanço material do petróleo em uma região em fase de exploração matura - Bacia do Recôncavo, Brasil', Tese de Doutorado, Universidade Federal do Rio de Janeiro. http://www.coc.ufrj.br/pt/tesesde-doutorado/152-2008/1146-luiz-felipe-carvalho-coutinho

Companhia de Pesquisa de Recursos Minerais 2006, GeoSGB - Dados, informações e produtos do serviço geológico do Brasil, Brasil, visualizado em 20 de dezembro 2020, <http:// geosgb.cprm.gov.br/>

Corrar, L J., Paulo, E. \& Dias Filho, J. M. 2007, Análise multivariada: para os cursos de administração, ciências contábeis e economia, Atlas, São Paulo. ISBN: 9788522447077

CPRM - vide Companhia de Pesquisa de Recursos Minerais.

Dean, W.E., Arthur, M.A. \& Claypool, G.E., 1986, 'Depletion of 13C in Cretaceous marine organic matter: Source, diagenetic, or environmental signal?', Marine Geology, vol. 70, no. 1-2, pp. 119-157. https://doi.org/10.1016/0025-3227(86)90092-7

Dembicki Jr, H. 2009, 'Three common source rock evaluation errors made by geologists during prospect or play appraisals', AAPG Bulletin, vol. 93, no. 3, pp. 341- 56. https://doi. org/10.1306/10230808076

Espitalié, J., Laporte, M., Madec, M., Marquis, F., Leplat, P., Paulet, J. \& Boutefeu, A. 1977, 'Méthode rapide de caractérization des roches mères, de leur potentiel pétrolier et de leur degré d'évolution', Revue de l'Institut Français du Petrole, vol. 32, no. 1, pp. 23-42. https://doi.org/10.2516/ogst:1977002

Euzébio, R.S., Reis, D.E.S., Brito, M.A.R.C., Bergamaschi, S., Martins, M.V.A. \& Rodrigues, R. 2016, 'Oil generation potential assessment and paleoenvironmental interpretation of Irati Formation (Lower Permian) in northwestern of Paraná Basin (Brazil)', Journal of Sedimentary Environments, vol. 1, no. 2, pp. 261-274. https://doi.org/10.12957/jse.2016.23388 
Figueiredo, A.M.F., Braga, J.A.E., Zabalaga, J.C., Oliveira, J.J., Aguiar, G.A., Silva, O.B., Mato, L.F., Daniel, L.M.F., Magnavita, L.P. \& Bruhn, C.H.L. 1994, 'Recôncavo Basin, Brazil: A prolific intracontinental Rift Basin' in S.M. Landon (ed.), Interior Rift Basins, American Association of Petroleum Geologists, Tulsa, pp. 157-203.

Gaglianone, P.C. \& Trindade, L.A.F. 1988, 'Caracterização geoquímica dos óleos da Bacia do Recôncavo', Geochimica Brasiliensis, vol. 2, no. 1, pp. 15-39. http://dx.doi.org/10.21715/ gb.v1i2.13

Guzzo, J.V.P. 1997, 'Estratigrafia integrada e Paleolimnologia de uma seção de idade Aratu (Eucretácio) da Bacia do Recôncavo, NE do Brasil', Dissertação de Mestrado, Universidade Federal do Rio Grande do Sul.

Hedges, J. I. \& Stern, J. H. 1984, 'Carbon and nitrogen determinations of carbonate-containing solids', Limnology and oceanography, vol. 29, no. 3, pp. 657-63. https://doi. org/10.4319/1o.1984.29.3.0657

Hunt, J. M. 1996, Petroleum Geochemistry and Geology, 2nd edn, Ed. Freeman, São Francisco. ISBN 0-7167-2441-3.

Lafargue, E., Marquis,F. \& Pillot, D. 1998, 'Les applications de Rock-Eval 6 dans l'exploration et la production des hydrocarbures, et dans les études de contamination des sols', Institut Français du Pétrole, vol. 53, no. 4, pp. 421-37. https:// doi.org/10.2516/ogst:1998036

Magnavita, L. P. 1992, 'Geometry and kinematics of the Recôncavo-Tucano_Jatobá Rift, NE Brazil', Tese de doutorado, Universidade de Oxford.

Magnavita, L. P., Silva, R. R. \& Sanches, C. P. 2005, 'Roteiros geológicos: Guia de Campo da Bacia do Recôncavo, NE do Brasil', Boletim de Geociências da Petrobras-Bacias rifte, vol. 13, no. 2, pp. 301-34.

Mello, M.R., Mohriak, W.U., Koutsoukos, E.A.M. \& Bacoccoli, G. 1994, 'Selected petroleum systems in Brazil' in L.B. Magoon \& W.G. Dow (eds.), The Petroleum System - from Source to Trap, American Association of Petroleum Geologists, Tulsa,pp. 499-512. https://doi.org/10.1306/M60585

Mello, M.R., Gaglianone, P.C., Brassell, S.C. \& Maxwell, J.R. 1988, 'Geochemical and biological marker assessment of depositional environments using Brazilian offshore oils', Marine and Petroleum Geology, vol. 5, pp. 205-23. https:// doi.org/10.1016/0264-8172(88)90002-5

Peters, K.E. 1986, 'Guidelines for evaluating petroleum source rock using programmed Pyrolysis', AAPG Bulletin, vol. 70, no. 3, pp. 318-29. https://doi.org/10.1306/94885688-170411D7-8645000102C1865D

Peters, K.E. \& Cassa, M.R. 1994, 'Applied source rock geochemistry' in L.B. Magoon \& W.G. Dow (eds), The Petroleum System - from Source to Trap. American Association of Petroleum Geologists, Tulsa, pp. 93-120. https://doi.org/10.1306/M60585C5
Peters, K.E. \& Moldowan, J.M. 1993, The Biomarker Guide Interpreting Molecular Fossils in Petroleum and Ancient Sediments, Prentice Hall, New Jersey. ISBN 0-13-086752-7

Pereira, A.L. 2007, 'Isótopos estáveis em estudos ecológicos: métodos, aplicações e perspectivas', Rev. Biociências, vol. 13 , no. $1-2$, pp. 16-27.

Portela, H. A. 2012, 'Caracterização da matéria orgânica potencialmente geradora de petróleo e seu posicionamento bioestratigráfico, com base em Palinologia e Palinofácies da Formação Pojuca- Bacia do Recôncavo', Tese Doutorado, Universidade Estatual do Rio de Janeiro.

Portela, H. A., Antonioli, L. Oliveira, R. M. A. G., Amaral, P. F. \& Dino, R. 2016, 'Análise de palinofácies na avaliação do potencial gerador da Formação Pojuca, bacia do recôncavo', Revista Brasileira de Paleontologia, vol. 19, no. 2, pp. 271-82. http://dx.doi.org/10.4072/rbp.2016.2.10

Rodrigues, G. B. \& Fauth, G. 2013, 'Isótopos estáveis de carbono e oxigênio em ostracodes do Cretáceo: metodologias, aplicações e desafios', Terra e didatica vol. 9, pp. 34-49. https://doi. org/10.20396/td.v9i1.8637408

Routh, J., Mcdonald, T. J. \& Grossman, E. L. 1999, 'Sedimentary organic matter sources and depositional environment in the Yegua formation (Brazos County, Texas)', Organic Geochemistry, vol. 30, no. 11, pp. 1437-53. https://doi. org/10.1016/S0146-6380(99)00118-7

Santos, C. F. 2005, 'Estratigrafia de Seqüências da fase final de preenchimento de um rifte intracontinental: um modelo com base no Barremiano Inferior da Bacia do Recôncavo' Boletim de Geociencias da Petrobras, vol. 13, no. 2, pp. 205-26.

Silva, O.B., Caixeta, J.M. \& Milhomem, P.S. 2007, 'Bacia do Recôncavo', Boletim de Geociências da Petrobras, vol. 15, no. 2, pp. 42331.

Tissot, B., Durand, B., Espitalie, J. \& Combaz, A. 1974, 'Influence of the nature and diagenesis of organic matter in the formation of petroleum', AAPG Bulletin, vol. 58, pp. 499 -506. https://doi.org/10.1306/83D90CEB-16C7-11D78645000102C1865D

Tissot, B.P. \& Welte, D.H. 1978, Petroleum Formation and Occurrence: A New Approach to Oil and Gas Exploration, Springer-Verlag, New York. ISBN 3540086986

Tissot, B. P., \& Welte, D. H. 1984, Petroleum formation and occurrence, Springer-Verlag, New York. ISBN 978-3-64287815-2

Zambrano, E.R.N., Oliveira, O.M.C. \& Ribeiro, H.J.P.S. 2017, 'Caracterização geoquímica com indícios paleoambientais de folhelhos da Formação Pimenteiras, estado do Tocantins, Bacia do Parnaíba, Brasil', Geologia USP - Série Cientifica, vol. 17, no. 3, pp. 67-78. http://dx.doi.org/10.11606/issn.23169095.v17-395

\section{Como citar:}

Miranda, F.L.C., Cerqueira, J.R., Amaral, D.N., Abreu, N.C., Góes, V.C.M., Silva, A.S., Dino, R., Antonioli, L., Queiroz, A.F.S., Santos, L.C.L. \& Garcia, K.S. 2021, 'Geoquímica Orgânica de Folhelhos da Formação Pojuca, Bacia do Recôncavo, Brasil', Anuário do Instituto de Geociências, vol. 44: 40847. https://doi.org/10.11137/1982-3908_2021_44_40847 